\section{ANALYSIS OF PARTICLE DISTRIBUTION IN A DOUBLE LAYER BEAM SHAPING ASSEMBLY RESULTED FROM 30 MEV-PROTON REACTIONS WITH BERYLLIUM TARGET USING THE PHITS PROGRAM}

Bilalodina,b*, Gede Bayu Supartaa, Arief Hermantoa, Dwi Satya Palupia, Yohannes Sardjonoc, Rasitod

aDepartment of Physics, Faculty of Mathematics and Natural Science, Gajah Mada University, Indonesia bDepartment of Physics, Faculty of Mathematics and Natural Science, Jenderal Soedirman University, Indonesia cCentre for Accelerator Science and Technology, National Nuclear Energy Agency, Indonesia ¿Center for Applied Nuclear Science and Nuclear Agency, Bandung, Indonesia
Article history

Received

17 October 2019

Received in revised form

24 February 2020

Accepted

12 April 2020

Published online

23 April 2020

*Corresponding author bilalodin@unsoed.ac.id

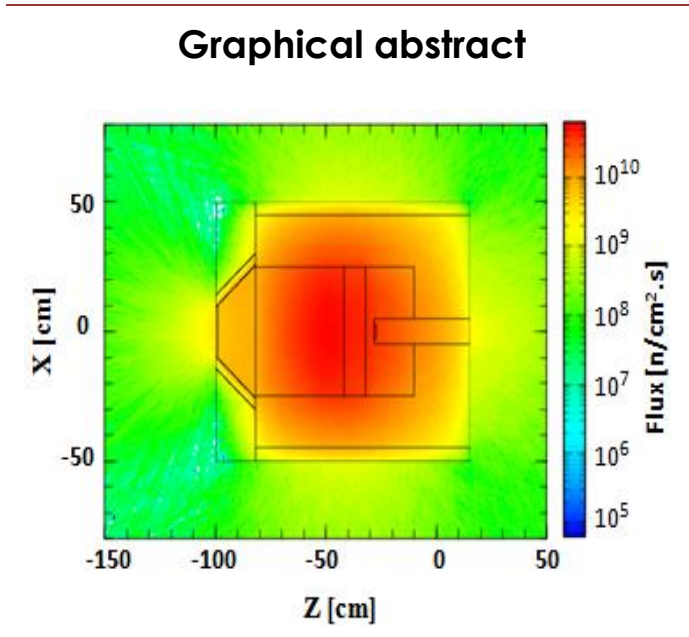

\begin{abstract}
An analysis on the distribution of particle flux emanating from reactions of 30 MeV-proton with beryllium target in a double layer beam shaping assembly (BSA) has been carried out using the PHITS program. It studies important parameters relating to the distribution of proton, neutron, and gamma. It is revealed that reactions of proton and beryllium in double layer BSA produce fast neutrons and other protons, resulting from certain reactions, and recoil protons from the interactions of fast neutrons and hydrogen atoms. Fast neutrons are distributed around beryllium target, moderator, reflector, and collimator. They are moderated by $\mathrm{Al}$ and LiF material. Epithermal neutrons spread along the moderator, with a distribution that is tapering down as it approaches the end of the collimator (aperture). During its travel along the moderator, an epithermal neutron decreases in energy to become a thermal neutron. The spectrum of neutron beam produced by the double layer BSA is wide, which indicates that the neutron beam exiting the aperture consists of three kinds of neutrons, dominated by epithermal neutrons with energy range $1 \mathrm{eV}-10 \mathrm{keV}$.
\end{abstract}

Keywords: Particle distribution, ${ }^{9} \mathrm{Be}(\mathrm{p}, \mathrm{n})^{9} \mathrm{~B}$ reaction, double layer $\mathrm{BSA}$, spectrum of neutron beam, PHITS program

(c) 2020 Penerbit UTM Press. All rights reserved

\subsection{INTRODUCTION}

The key to a successful cancer therapy using the Boron Neutron Capture Therapy (BNCT) methods is determined by, among others, two factors, specifically boron compound accumulated in cancer cells and availability of neutron sources [1]. One of the neutron sources for BNCT purposes is the cyclotron [2]. A neutron producing reaction in the cyclotron is the reaction of $30 \mathrm{MeV}$ protons with the 
${ }^{9}$ Be target. However, the characteristic of the resulting neutrons does not allow direct applications because apart from fast neutrons, contaminants are also produced. Neutron beams remain to be processed using the beam shaping assembly (BSA) system $[3,4]$.

The Components of a BSA are moderator, reflector, collimator, and filter. The functions of each component of BSA are as follows. The moderator serves to lower the energy of fast neutrons into that of epithermal neutrons. The reflector is for increasing neutron flux and reducing its leak. The collimator is for focusing the beam, and the filter is for suppressing gamma radiations and non-epithermal neutrons [5].

Each of BSA components is commonly designed with single layer configuration, i.e., they only use one type of material. The downside of the single layer configuration is that every component does not work efficiently, causing the moderated fast neutrons to not be transformed into epithermal neutrons with such quality and intensity that agree with the requirements of $\mathrm{BNCT}$ therapies. To overcome the weakness, double layer, even multilayer, configurations may be developed.

Evidence suggests that combining two suitable materials yields better quality. Using two-layered moderators combination, Dao-wen et al., (2012) [6] were able to improve moderation up to $19.3 \%$. Adib et al., (2016) [7] combined two or more filter materials and succeeded to produce epithermal neutron beams within a range of 1.5 to $10 \mathrm{keV}$. Better results were also obtained when two reflectors are combined. A combination of reflector materials such as tungsten (W) and molybdenum (Mo) can multiply neutron reflection to five times as much, compared to when only tungsten is used $[5,8]$. Based on these evidences, the use of four BSA components in the form of a double layer configuration is expected to produce epithermal neutron beams with better intensity and quality.

Until now visualizations regarding interacting mechanism between particles and component materials are hardly ever conducted. This is contrary to the fact that visualization is useful to know the distribution of interacting particles with matter. The use of the MCNP programming to display particle distributions in BSA is deemed inefficient considering its complex procedure, particularly with regards to mesh tally and graphical output display. By contrast, the PHITS programming is regarded to have easier procedure in graphical output display [9, 10]. This article discusses an analysis of the distribution of particles interacting with BSA materials using the PHITS programming. The BSA of interest is that of a double layer using two kinds of materials [11].

\subsection{METHODOLOGY}

The double layer BSA model used in this simulation consists of four typical components: moderator, filter, reflector, and a collimator. The material in the moderator is be made of Aluminum (Al) and lithium fluoride (LiF). The material for fast and thermal neutron filter are FeC (Carbon Iron) and Cadmium (Cd), whereas the collimator is made of Nickel and borated polyethylene. A Lead (Pb) material for gamma shielding is commonly attached to the end of BSA. The neutrons are modeled to come from interactions between $30 \mathrm{MeV}$ proton and beryllium (Be) target, at $1 \mathrm{~mA}$ proton current [12]. In this model, neutron flux is calculated using the following Equations [13].

$$
\begin{gathered}
\varphi_{\mathrm{n}}=\mathrm{N} \sigma \varphi_{\mathrm{p}} \\
N=\frac{V \rho N_{A}}{B_{A}} \\
\varphi_{p}=\frac{I}{e A}
\end{gathered}
$$

where $V$ is the volume of $B e, \rho$ is the density of $B e, N_{A}$ is the Avogadro number, $B_{A}$ is the atomic weight of $\mathrm{Be}, \mathrm{l}$ is proton current ( $1 \mathrm{~mA})$, e is elementary charge, $A$ is the area of $B e$ and $\sigma$ is macroscopic cross section.

Based on calculation using Equation 1 it is obtained that the neutron flux is $1.48 \times 10^{12} \mathrm{n} / \mathrm{cm}^{2} . \mathrm{s}$.

The distribution of protons, neutrons, and gamma fluxes in the BSA and the energy spectrum of the resulting neutrons are studied using the PHITS program. The distribution of particles is computed using track tally. The calculation of gamma dose also uses track tally along with flux to dose converter. Particle trajectories and geometries are visualized using the ANGEL software. The library data of cross sections for neutron and photon is obtained from the JENDL-4.0, while for proton is from the intra-nuclear cascade (INCL4.6) [9, 10]. The design of the double layer BSA and the research flowchart are shown in Figure 1 and Figure 2, respectively.

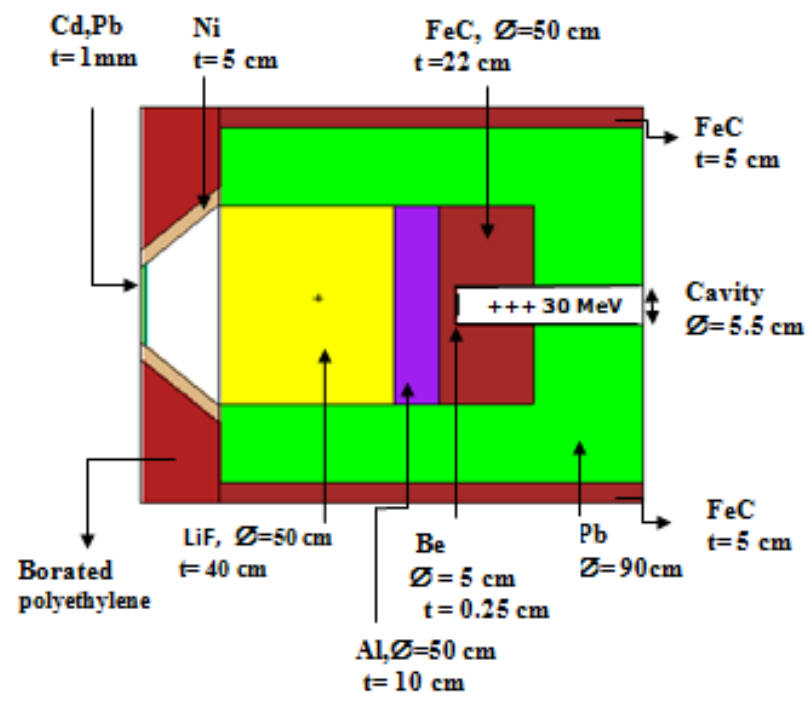

Figure 1 The design of double layer BSA 


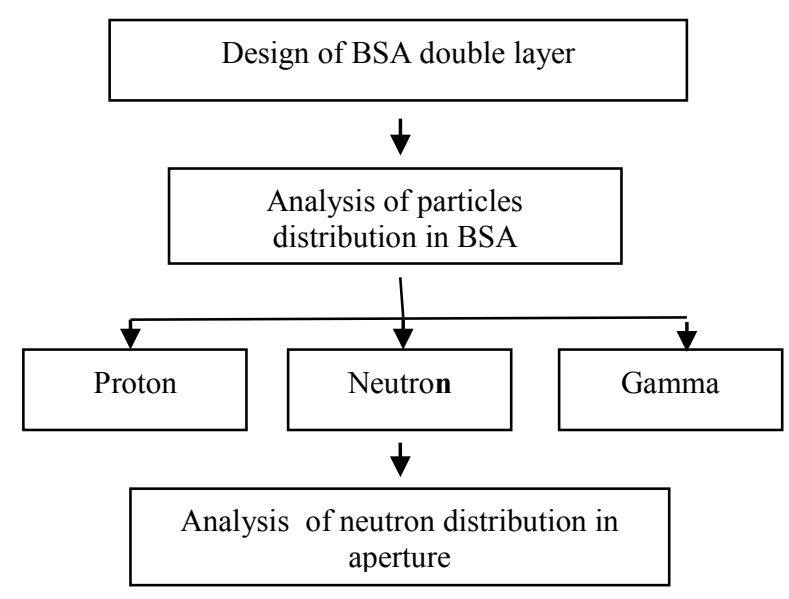

Figure 2 The research flowchart of the stage in determining particle distribution in the BSA

\subsection{RESULTS AND DISCUSSION}

\subsection{Distribution of Protons}

Computational results about the distribution of particles in the double layer BSA using the PHITS programming are shown in the following figures. The distribution of proton, neutron, and gamma photon fluxes are shown in colors ranging from blue to red. Blue and red represent the lowest flux and the highest flux, respectively.

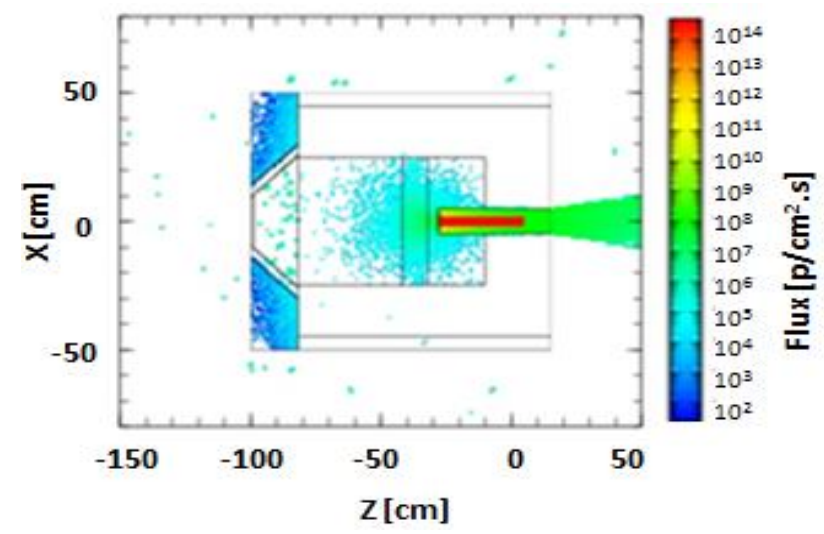

Figure 3 Distribution of proton in BSA

Figure 3 shows the proton flux in the double layer BSA. The red color represents protons from accelerator having the energy of $30 \mathrm{MeV}$, while the blue color indicates new protons around the target, the moderator, and the collimator. It suggests that during their interactions with beryllium, only partial numbers of the protons become neutrons. The intensity of protons in the vicinity of beryllium target is $10^{4} \mathrm{p} / \mathrm{cm}^{2}$.s. New protons in the neighborhood of the target and moderator are resulted from ${ }^{9} \mathrm{Be}(p, x n)$ reactions [14]. The protons surrounding the collimator come from interactions of fast neutrons with hydrogen atoms of borated polyethylene material through elastic scattering reaction [15]. The flux of such protons is $10^{2} \mathrm{p} / \mathrm{cm}^{2} . \mathrm{s}$

\subsection{Distribution of Neutrons}

This simulation shows that the interactions of protons with beryllium target are able to produce maximum neutron yield of $10^{14}(\mathrm{n} / \mathrm{s} \mathrm{mA})$ and average total flux of $10^{12} \mathrm{n} / \mathrm{cm}^{2}$.s. The neutrons are mainly resulted from reactions of $30 \mathrm{MeV}$ protons with ${ }^{9} \mathrm{Be}$ target material through ${ }^{9} \mathrm{Be}(p, n)^{9} \mathrm{~B}$ reaction $[3,16]$. By calculation using empirical equations, such reactions produce fast neutrons with the maximum energy of $28 \mathrm{MeV}$ [3] and average energy of $11.9 \mathrm{MeV}$ [17].

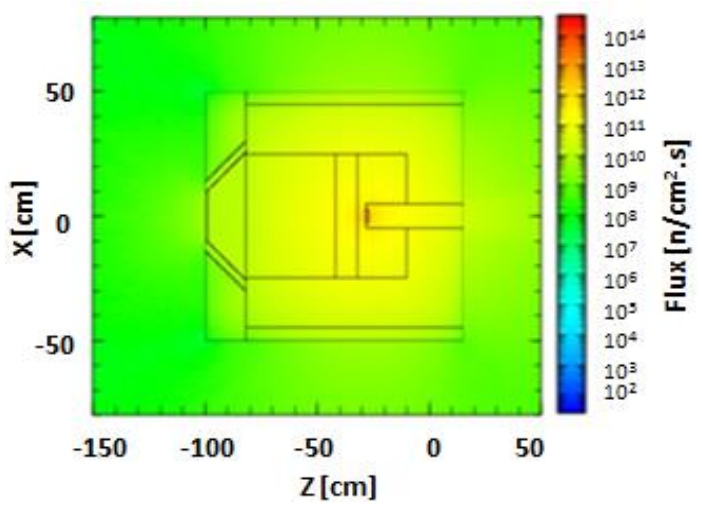

Figure 4 Distribution of fast neutron flux

Figure 4 shows the distribution of fast neutrons in the double layer BSA. The highest distribution of fast neutron is found in the vicinity of the beryllium target, indicated in red color. Fast neutrons spread across all components of the BSA, i.e. moderator, reflector, and collimator, which is shown in yellow color. The flux of fast neutrons going through the moderator changes as indicated by the change in color from yellow to green. The color change indicates there is a decrease in fast neutron flux due to fast neutrons being moderated by $\mathrm{Al}$ and LiF, and then transformed into epithermal and thermal neutrons. The flux of fast neutron as well as the intensity also decreases after going through $\mathrm{Pb}$ and $\mathrm{FeC}$ reflector, indicated by color change from yellow to green. such flux and intensity decrease confirms that the reflector effectively reflects fast neutrons back into the moderator [18].

Although $\mathrm{Pb}$ reflector and carbon-iron $(\mathrm{FeC})$ are able to reflect fast neutrons, some fast neutrons are still able to escape from the reflector. Such an occurrence may be due to the reflector wall thickness is still inadequate. Therefore, the reflector fails to prevent neutron current leaks [3].

Fast neutrons that are going through moderator and reflector will enter the collimator and undergo reflections at the collimator walls. The flux of fast neutrons resulted at the end of the collimator is $10^{7} \mathrm{n} / \mathrm{cm}^{2} . \mathrm{s}$. 


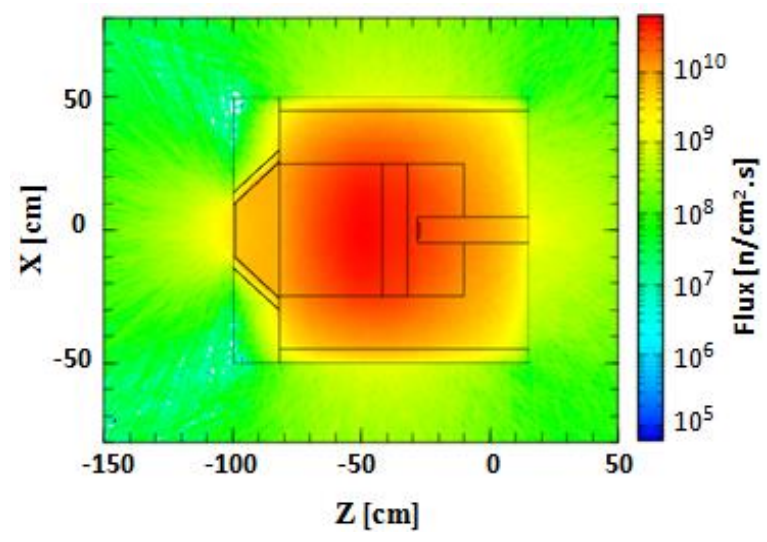

Figure 5 Distribution of the epithermal neutron flux

Figure 5 shows the distribution of epithermal neutrons after the moderation by $\mathrm{Al}$ and LiF materials in the double layer BSA. The highest distribution is found surrounding the moderator, as shown in red color. The effectiveness of $\mathrm{Al}$ and LiF in producing high epithermal neutron flux depends primarily on $\mathrm{Al}$ since it is has large scattering cross section with respect to neutrons with the energy of above $10 \mathrm{keV}$ [19]. The interactions of neutrons with Al material are predominantly through ${ }^{27} \mathrm{Al}(\mathrm{n}, 2 \mathrm{n})^{26} \mathrm{Al}$ reactions, producing epithermal neutrons [20]. The existence of fluorine (F) element in LiF material enhances fast neutron moderations since fluorine has large scattering cross section with respect to fast neutrons. Hence, LiF contributes to increasing the number of epithermal neutrons $[19,21]$. Epithermal neutron flux surrounding the moderator is around $10^{11} \mathrm{n} / \mathrm{cm}^{2} . \mathrm{s}$.

Epithermal neutrons are also distributed inside the components of the BSA, i.e. reflector and collimator. In the first reflector the intensity of epithermal neutron is relatively high, as indicated by the red color, but it decreases in the second reflector as indicated by yellow color. Such high intensity of epithermal neutron flux in the first reflector may be caused by fast neutrons being reflected back into the moderator, in which some of them turn into epithermal neutrons. As a result, the intensity of epithermal neutron flux in the BSA increases. The intensity decrease of epithermal neutron flux in the second reflector may be due to some epithermal neutrons turning back into thermal neutrons [18]. However, the intensity of epithermal neutron flux outside the wall of reflector is still high. Therefore, optimization in thickness of the reflector is needed to reduce the loss of epithermal neutron flux.

At the end of the collimator the intensity of epithermal neutron flux also decreases, as shown by color change from red to yellow. We found the value of epithermal neutron flux at the end of the collimator is about $10^{9} \mathrm{n} / \mathrm{cm}^{2} . \mathrm{s}$.

Figure 6 shows the distribution of thermal neutron flux in the double layer BSA. The highest distribution of thermal neutron flux is found in the moderator component, shown in red color. Such high intensity of thermal neutron in the moderator may be resulted from two mechanisms. First, thermal neutrons are formed when epithermal neutrons are transformed into thermal neutrons, and second, epithermal neutrons move through the first reflector $[11,16]$.

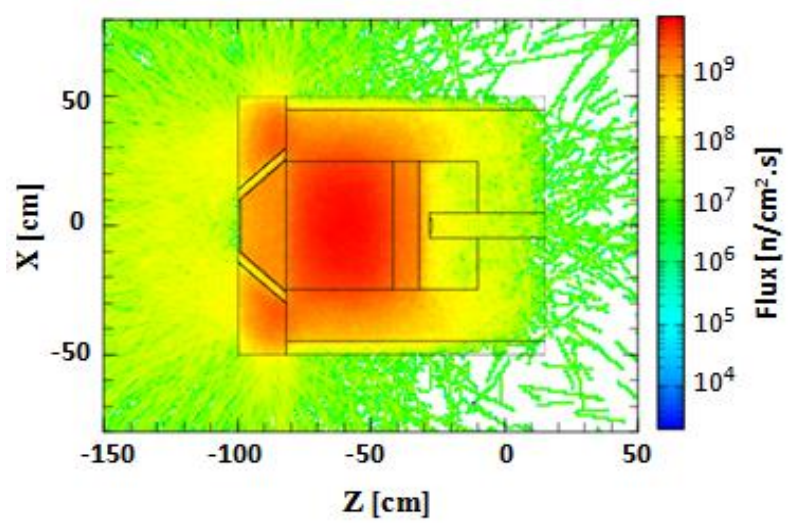

Figure 6 Distribution of the thermal neutron flux

The intensity of thermal neutron at the collimator wall is also high as it shown in red color. It is caused by the interaction of fast neutrons with $\mathrm{H}$ atom in borated polyethylene material [22]. We found that the intensity of thermal neutron flux after going through Cd filter decreases to $10^{6} \mathrm{n} / \mathrm{cm}^{2} . \mathrm{s}$.

The intensity of thermal neutron outside the reflector is sufficiently low, as it shown in green and white color. $\mathrm{Pb}$ and $\mathrm{FeC}$ are able to significantly suppress thermal neutron flux.

\subsection{Distribution of Gamma Particle}

Figure 7 shows the distribution of gamma rays in the BSA shown in yellow color. We found the flux of gamma rays around the beryllium target is $10^{10} \gamma / \mathrm{cm}^{2}$.s. The gamma rays are produced mainly from interactions of protons with beryllium target through ${ }^{9} \mathrm{Be}(\mathrm{p}, \alpha)^{6} \mathrm{Li}(\gamma)$ reactions. A small number of gamma rays are also emitted from capture reaction followed by inelastic scattering through ${ }^{9} \mathrm{Be}(\mathrm{p}, \gamma)^{10 \mathrm{Be}}$ reactions [23]. Gamma rays are also produced by the reaction of neutrons with aluminum through ${ }^{27} \mathrm{Al}(\mathrm{n}, \gamma)^{28} \mathrm{Al}$ reactions[20]. The flux of gamma rays declines after leaving the collimator.

To obtain a neutron beam that is suitable for $\mathrm{BNCT}$, fast neutron, thermal neutrons and gamma rays need to be filtered. FeC material is best in decreasing fast neutron. The effectiveness of Fe as fast neutron filter is due to its ability to scatter neutrons in-elastically as they pass through $\mathrm{Fe}$ material with the energy of higher than $14 \mathrm{MeV}$ [8]. Lead is an excellent gamma ray filters, as indicated by good photon attenuation [21]. To eliminate thermal neutrons, cadmium (Cd) is used, since $\mathrm{Cd}$ is a material with large thermal energy rate absorption cross section without absorbing significant parts of epithermal neutrons [8]. So, with $\mathrm{Pb}$ and $\mathrm{Cd}$ material 
as the last part of the BSA, the design can improve the BSA configuration in lowering contamination dose in patients and preventing healthy cells and tissues from damage.

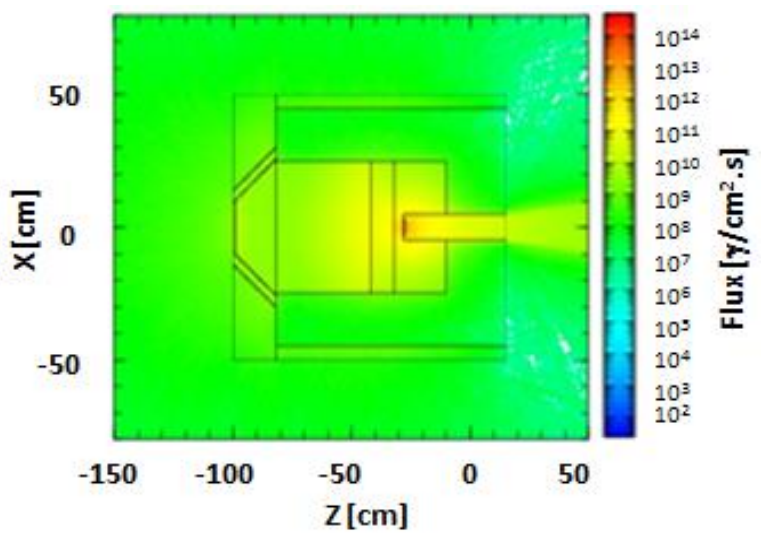

Figure 7 Distribution of gamma rays in the BSA

\subsection{Characteristics of Neutron Beam Spectrum}

The characteristic spectrum of neutron beam computed at the aperture of this BSA double layer design is shown in Figure 8. The spectrum is quite wide, specifically the neutron beams coming out from the aperture comprises three kinds of neutrons, which are thermal, epithermal and fast neutrons. Considering that the curve peaks at epithermal energy range (leV - $10 \mathrm{keV})$, the neutron beam coming out of aperture is dominated by epithermal neutrons. The spectrum of neutron beams calculated at the location of the aperture is characterized by the epithermal neutron flux of $1.0 \times 10^{9} \mathrm{n} / \mathrm{cm}^{2} . \mathrm{s}$, the ratio of epithermal to the thermal of 102 , and the ratio of gamma dose to the epithermal neutron flux of $5.75 \times 10^{-13} \mathrm{~Gy} . \mathrm{cm}^{2}$. Such energy is enough for deeply located cancers in BNCT therapies [24].

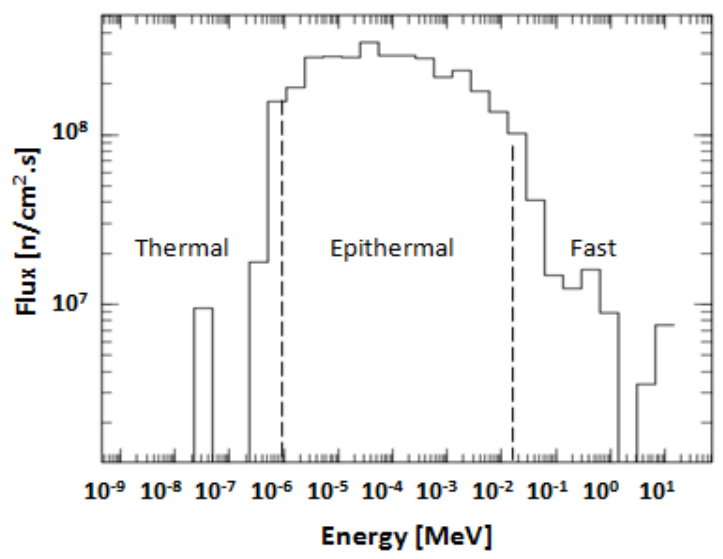

Figure 8 Spectrum of neutron beams coming out of the end of BSA (aperture)

\subsection{CONCLUSION}

Interactions of $30 \mathrm{MeV}$ proton beams with beryllium in double layer BSA produce fast neutrons. The interactions also produce proton by-products from a particular reaction and recoil protons, which appear from interactions of the fast neutrons with hydrogen atoms. Fast neutrons are distributed over beryllium target, moderator, and reflector. Fast neutrons are moderated into epithermal neutrons. Epithermal neutrons are distributed along the moderator and decrease in density toward the end of the collimator. Epithermal neutron flux computed at the aperture's position is $10^{9} \mathrm{n} / \mathrm{cm}^{2}$.s. During their passage in the moderator, some epithermal neutrons experience decreases in energy turning them into thermal neutrons. The spectrum of neutron beams produced by the double layer BSA displays a wide curve. This indicates that the neutron beam that leaves the end of the collimator (aperture) consists of three kinds of neutrons, being dominated by epithermal neutrons with the energy range of $1 \mathrm{eV}-10 \mathrm{keV}$.

\section{Acknowledgment}

We thank the Ministry of Research, Technology and Higher Education for its BPPDN Scholarships and BATAN for its consent to use the MCNPX and PHITS software, which allow this research to be conducted. We also thank the late Prof. Dr. Kusminarto for his support in conducting the research.

\section{References}

[1] Sauerwein, W. A. G. 2012. Neutron Capture Therapy. New York: Springer.

[2] Hashimoto, Y., Hiraga, F., Kiyanagi, Y. 2014. Effects of Proton Energy on Optimal Moderator System and Neutron-induced Radioactivity of Compact Accelerator-driven ${ }^{9} \mathrm{Be}(\mathrm{p}, n)$ Neutron Sources for BNCT. Physics Procedia. 60: 332-340.

Doi: https://doi.org/10.1016/j.phpro.2014.11.045.

[3] Tanaka, H., Sakurai, Y., Suzuki, M., Masunaga, S. Matsumoto, T., Fujita, K., Kashino, G., Kinashi, Y., Liu, Y., Takada, M., Ono, K., dan Marushasi. 2011. Experimental Verification of Beam Characteristics for Cyclotron-based Epithermal Neutron Source (C-BENS). Applied Radiation and Isotopes. 69 (12): 1642-1645.

Doi: 10.1016/j.apradiso.2011.03.020.

[4] Monshizadeh, M., Kasesaz, Y., Khalafi, H. S., Hamidi. 2015 MCNP Design of Thermal and Epithermal Neutron Beam for BNCT at the Isfahan MNSR. Progress in Nuclear Energy. 83: 427-432.

Doi: https://doi.org/10.1016/j.pnucene.2015.05.004.

[5] Kasesaz, Y., Khalafi, H., Rahmani, F. 2013. Optimization of the Beam Shaping Assembly in the D-D Neutron Generators-based BNCT using the Response Matrix Method. Applied Radiation and Isotopes. 82: 55-59 Doi: https://doi.org/10.1016/j.apradiso.2013.07.008.

[6] Dao-wen, C., Jing-bin, L., Dong, Y., Hui-dong, W., Ke-yan, M. 2012. Improvement of the Moderator's Thermalization Efficiency for $14 \mathrm{MeV}$ Neutrons in Boron Neutron Capture Therapy. Journal of Radioanalytical and Nuclear Chemistry. 292(3): 1085-1088. 
Doi: 10.1007/s10967-011-1575-z.

[7] Adib, M., Habib, N., Bashter, I. I., El-mesiry, M. S., \& Mansy, M. S. 2016. Simulation Study of Accelerator Based Quasimono-energetic Epithermal Neutron Beams for BNCT. Applied Radiation and Isotopes. 107: 98-102. Doi: https://doi.org/10.1016/j.apradiso.2015.10.003.

[8] Asnal, M., Liamsuwan, T., Onjun, T. 2015. An Evaluation on the Design of Beam Shaping Assembly Based on the D-T reaction for BNCT. Journal of Physics: Conference Series. 611: 1-7.

Doi: 10.1088/1742-6596/611/1/012031.

[9] Sato, T., Niita, K., Matsuda, N., Hashimoto, Iwamoto, Y., Noda, S., ... \& Okumura, K. 2013. Particle and Heavy Ion Transport code System, PHITS, version 2.52. Journal of Nuclear Science and Technology. 50(9): 913-923. Doi: https://doi.org/10.1080/00223131.2013.814553.

[10] Sato, T., Niita, K., Matsuda, N., Hashimoto, S., Iwamoto, Y., Furuta, T., ... \& Fukahori, T. 2015. Overview of Particle and Heavy lon Transport Code System PHITS. Annals of Nuclear Energy. 82: 110-115.

Doi: https://doi.org/10.1016/j.anucene.2014.08.023.

[11] Bilalodin, Kusminarto, Hermanto, A., Sardjono,Y. 2017. Double Layer Collimator for BNCT Neutron Source Based on $30 \mathrm{MeV}$ Cyclotron. Indonesian Journal of Physic and Nuclear Application. 3(2): 124-127.

Doi: https://doi.org/10.24246/ijpna.v2i3.124-127.

[12] Mitsumoto, T., Fujita, K., Ogasawara, T., Tsutsui, H., Yajima, S., Maruhashi, A., ... \& Tanaka, H. 2010. BNCT System using $30 \mathrm{MeV}$ HM-30 Cyclotron. Proceeding of Cyclotron. 6-8.

[13] Sunardi and Silakhuddin. 2015. Estimation of Exposure Quantity of Gamma and Neutron in $13 \mathrm{MeV}$ Proton Cyclotron for Radio Isotope Production of ${ }^{18} \mathrm{~F}$. Proceedings of the Meeting and Scientific Presentation of Accelerator Technology and its Applications. 17: 2732.

www.iaea.org/inis/collection/NCLCollectionStore/_Publi c/47/082/47082696.pdf.

[14] Avagyan, R., Avetisyan, R., Ivanyan, V., Kerobyan, I. 2017. Geant4 Simulations of a Beam Shaping Assembly Design and Optimization for Thermal/epithermal Neutrons. Acta Physica PolonicaB. 48(10): 1693-1699.

Doi: 10.5506/APhysPolB.48.1693.

[15] Salehi, D., Sardari, D., Salehi, M. 2012. Evaluation of Design Neutron Filters in BNCT. Science and Research Branch. Islamic Azad University Tehran, Iran, Open Access Open Access Scientific report. 1 (11): 2-6. Doi: http:/dx.doi.org/10.4172/scientificreport.537.
[16] Sato, A., Takizawaa, Y., Hiragaa, F., Kiyanagia, Y. 2014. Neutron Slowing Down Efficiency Depending on the Proton Energy for Accelerator based BNCT. Physics Procedia. 60: 15-22.

Doi: https:/ doi: 10.1016/j.phpro.2014.11.004.

[17] Stefanik, M., Bem, P., Majerle, M., Novak, J., Simeckova E., \& Stursa, J. 2019, Neutron Field Study of p (35)+ Be Source Reaction at the NPI Rez. Radiation Physics and Chemistry. 155: 294-298.

[18] Takata, T., Tanaka, H., Sakurai, Y., \& Maruhashi, A. 2010. Increase in Irradiation Beam Intensity by Using a Hybrid Target System in Cyclotron-based Neutron Capture Therapy. Journal of Nuclear Science and Technology. 47(7): 575-581

Doi: http:/dx.doi.org/10.1080/18811248.20109720954.

[19] Osawa, Y., Imoto, S., Kusaka, S., Sato, F., Tanoshita, M., \& Murata, I. 2017. Development of an Epi-thermal Neutron Field for Fundamental Researches for BNCT with a D-T Neutron Source. EPJ Web of Conferences. 153: 1-9. Doi: https://doi.org/10.1051/epjconf/201715304008.

[20] Ma, W. C., Zhang, Q. G., Wang, W. H., Zuo, J. X. 2015 Neutron-induced Reactions on AlF3 Studied using the Optical Model. Nuclear Instruments and Methods in Physics Research B. 356-357: 42-45. Doi: https://doi.org/10.1016/j.nimb.2015.04.060.

[21] Ivakhin, V. S., Tikhomirov, V. G., Bolozdynya, I. A., Akimov, A. G., Stekhanov, V. N. 2011. Modeling of Filters for Formation of Mono-energetic Neutron Beams in the Research Reactor IRT MEPhl. Proceedings of GLOBAL. Makuhari, Japan. Paper No. 392341.

[22] Subramanian, D. V., Haridas, A., Kumar, D. S., Arul, A. J., \& Puthiyavinayagam, P. 2018. Neutron Attenuation Studies with Borated Polyethylene Slabs Containing 30\% Natural Boron and Its Comparison with Hydrogenous Materials. Indian Journal of Pure \& Applied Physics. 56: 583-586.

Doi: http://nopr.niscair.res.in/handle/123456789/44863.

[23] Hu, G., Hu, H. S., Wang, S., Pan, Z, H., Jia, Q. G., Yan, M. F. 2016. The "Neutron Channel Design"-A Method for Gaining the Desired Neutrons. AIP Advances. 6: 1-12. Doi: https://doi.org/10.1063/1.4972203. Doi: 10.1088/1742-6596/611/1/012031.

[24] Faghihi, F., \& Khalili, S. 2013. Beam Shaping Assembly of a D-T Neutron Source for BNCT and Its Dosimetry Simulation in Deeply-seated Tumor. Radiation Physics and Chemistry. 89: 1-13.

Doi: https://doi.org/10.1016/j.radphyschem.2013.02.003. 